\title{
Monitorización de las cargas de entrenamiento en corredores de fondo y medio fondo de alto nivel
}

Miguel Pascual' , Marta Leyton'2, José Oriol ${ }^{1}$ y Marco Batista ${ }^{3}$

IUniversidad Autónoma de Madrid; 2Universidad Fundación San Pablo CEU de Sevilla; ${ }^{3}$ Instituto Politécnico de Castelo Branco (Portugal)

Email: miguel.pascualh@estudiante.vam.es

RESUMEN: El principal objetivo del entrenamiento consiste en mejorar el rendimiento y, por ende, reducir lesiones. Para ello, la monitorización de dicha carga (interna, externa y su relación) se ha constatado como vital. Sin embargo, muchos entrenadores siguen sin controlar (o etéreamente) las cargas en sus deportistas, pues, además, no existe un sistema etiquetado como el ideal. Consecuentemente, teniendo en cuenta el contexto y lo que la literatura dice al respecto, se realizó una propuesta personal con corredores de resistencia de alto nivel, contabilizando, en cuanto a carga externa, volumen mediante los kilómetros recorridos semanalmente, intensidad mediante la velocidad de carrera con zonas de entrenamiento en función del perfil individual de lactato, y frecuencia registrando el número de entrenamientos y de los contenidos trabajados. Respecto al estrés interno, la Escala de Esfuerzo Percibido y la medición de la altura del salto vertical con contramovimiento, en pos de determinar la fatiga neuromuscular subaguda. Los resultados mostraron relación significativa en la mayoría de variables, haciendo que el objetivo e hipótesis que perseguía la intervención se cumplan en mayor o menor medida, por lo que el sistema de monitorización escogido y/o los diferentes ítems que lo componen podrían ser válidos para su utilización en otros contextos.

PALABRAS CLAVE: monitorización, carga interna, carga externa, fatiga, deportes de resistencia.

\section{Monitoring of training loads in high-level middle and long-distance runners}

ABSTRACT: The main objective of training is to improve performance and, therefore, reduce injuries. For that reason, the monitoring of this load (internal, external and their relationship) has been verified as vital. However, many trainers still do not control (or ethereally) the loads on their athletes. In addition, there is not system labeled as the ideal. Consequently, taking into account the context and what the literature says about it, a personal research was made with high-level resistance runners, accounting, in terms of external load, volume through the kilometers traveled weekly, intensity through the running speed with training zones depending on the individual lactate profile, and frequency recording the number of training sessions and the contents worked. Regarding internal stress, the Rating of Perceived Exertion and a measurement of countermovement jump height, in order to determine subacute neuromuscular fatigue. The results showed a significant relationship in most variables, making the objective and hypothesis of the intervention have been met to a greater or lesser extent, so that the chosen monitoring system and / or the different items that comprise it could be valid for its use in other contexts.

KEY WORDS: monitoring, internal load, external load, fatigue, endurance sports. 


\section{INTRODUCCIÓN}

En el ámbito deportivo, el proceso del entrenamiento es crucial a la hora de conseguir una serie de adaptaciones específicas en el organismo que permitan conseguir un mayor rendimiento y, por consiguiente, unos mejores resultados en la competición. Sin embargo, el quiz de la cuestión está en saber cuál es la carga necesaria que requiere cada deportista en cada momento de su temporada en particular y de su carrera deportiva en general, con el fin de alcanzar su máximo potencial, sin llegar a sobrepasar su umbral de toleración.

A razón de todo ello, parece, entonces, claro que, un aspecto fundamental en todo este transcurso debiera de ser la monitorización de las cargas de entrenamiento, pues el llevar de forma acertada este procedimiento ayuda a analizar y establecer relaciones causales entre el entrenamiento, el rendimiento y las adaptaciones fisiológicas (Mujika, 2013), determinando si un deportista se está adaptando correctamente al programa de entrenamiento $\mathrm{y}$, de esta manera, reduciendo el riesgo de desarrollar sobrecarga no funcional, enfermedad y/o lesión (Halson, 2014). O, dicho de otra manera, los aumentos al azar en el volumen, intensidad y frecuencia, así como en la recuperación de los entrenamientos, incrementan la probabilidad de lesión y de aparición de los síntomas de sobreentrenamiento (Borresen y Lambert, 2009; O'Connor, 2007).

No obstante, la realidad es que, aún muchos entrenadores y/o preparadores físicos siguen sin controlar (o monitorizan, pero de manera etérea y subjetiva) las cargas a las cuales someten a sus deportistas (Jiménez-Reyes, Cuadrado-Peñafiel, y González-Badillo, 2011) con todo lo que ello supone. Asimismo, dicho inconveniente se ve incrementado, entre otras razones, debido a que son multitud los métodos que se pueden usar (Borresen y Lambert, 2008, 2009; Cejuela-Anta y Esteve-Lanao, 2011; Esteve Lanao, 2007; Mujika, 2006, 2017), pero ninguno de ellos ha alcanzado el nivel de ideal (Halson, 2014). Esto último se debe a que, por una parte, sus efectos sobre el organismo pueden diferir en función del tipo de individuo y, por otra, que aún existen numerosos problemas (metodológicos e instrumentales) para poner en funcionamiento ciertas prácticas (Balsalobre-Fernández, 2015; Wallace, Slattery, Impellizzeri, y Coutts, 2014).

De esta manera, algunos de los métodos/indicadores que más se han utilizado (y utilizan) en deportes de resistencia son: 1) En cuanto a la variable volumen, el cálculo del tiempo de trabajo o de la distancia recorrida. 2) Respecto a la frecuencia, la contabilización del número de sesiones por unidad de tiempo y/o de las sesiones específicas de un contenido en concreto por unidad de tiempo. 3) Por último, es respecto a la variable intensidad donde más heterogeneidad y controversias se puede encontrar en la literatura, como consecuencia, principalmente, de su mayor aspecto cualitativo. A razón de ello, nos podemos encontrar procedimientos subjetivos, como las escalas, los cuestionarios, los diarios o la mera observación directa (aunque éstos también pueden servir para monitorizar la frecuencia y volumen u otros ítems objetivos de intensidad), o indicadores objetivos, mediante marcadores fisiológicos (frecuencia cardiaca, lactato en sangre, o consumo de oxígeno, mayoritariamente) o mediante otros tipos de marcadores, destacando la velocidad de carrera o la producción de potencia. Luego, para mayor complejidad, dentro de cada uno de dichos indicadores se derivan otros. Por ejemplo, dentro de la frecuencia cardiaca se puede utilizar la FC absoluta, el \% FC máxima, el \% FC de reserva, la FC de recuperación, la variabilidad de la FC, etc.

Asimismo, a partir de las anteriores variables se han ido desarrollando diferentes índices para cuantificar la carga de entrenamiento/estrés en deportes de 
resistencia. Algunos de los más conocidos son: los métodos TRIMP (Training Impulse), siendo el precursor de ellos Banister y Calvert (1980), el TSS (Training Stress Score) cuyo algoritmo matemático fue desarrollado por Coggan (2003), las Zonas de entrenamiento, las cuales se pueden establecer a partir de numerosos indicadores, o el RPE de la sesión (RPE session) creado por Foster (1998) [texto borrado].

Asimismo, todos estos indicadores de la carga de entrenamiento y sus variables se pueden dividir, a su vez, en dos grandes grupos: los de carga externa y los de carga interna. La primera hace alusión al trabajo realizado por el atleta y medido independientemente de sus particularidades internas (Wallace, Slattery, y Coutts, 2009), mientras que la segunda se puede concretar como el relativo estrés fisiológico y psicológico que, individualmente, le ha supuesto al deportista un estímulo (Halson, 2014). A partir de aquí, entra en escena un concepto muy importante que va de la mano del atleta en todo el proceso del entrenamiento y las adaptaciones que se generan. Dicha concepción es la de la "fatiga", definida como la incapacidad o la necesidad de aumentar el esfuerzo por encima de lo habitual para llegar y/o mantener una intensidad de ejercicio demandada, lo cual puede ser considerado como una señal de alarma del organismo que indica una situación de estrés que disminuye las capacidades lógicas del individuo (Petibois, Cazorla, Poortmans, y Déléris, 2002).

Por esta razón, parece ser que la mejor opción para llevar a cabo el monitoreo del entrenamiento es aquel que combina ambos tipos de carga, pues de esta forma, correlacionando la carga externa e interna, se puede llegar a revelar la fatiga en el deportista. Es decir, el desacoplamiento o no de las cargas externas e internas puede ayudar a diferenciar si un atleta está fresco o fatigado (Halson, 2014) y/o si el atleta está ganando o perdiendo aptitud física (Bourdon et al., 2017).

Dicho lo cual, si bien es verdad que hay ciertos aspectos que aún no se conocen con exactitud en el fenómeno de la fatiga, lo que sí se sabe es que, el tipo, duración e intensidad de los esfuerzos realizados, afecta a todo este entramado (Fitts, 1994) (he aquí otro de los grandes motivos para llevar un seguimiento de las cargas de entrenamiento) y que en última instancia, sea cual sea su causa principal (fisiológica, psicológica, etc.), lo que genera es una minoración para generar fuerza (Allen, Lamb, y Westerbland, 2008).

En virtud de ello, se debe destacar la cada vez más aceptada utilización de la evaluación de la función neuromuscular para monitorizar las cargas de trabajo y su correspondiente fatiga. Sin entrar en muchos más detalles, destacar que, dicha evaluación se puede establecer de diferentes formas, como pruebas de salto, en las que destacan el uso del salto con contramovimiento (CMJ, por sus siglas en inglés Countermovement Jump) y sin contramovimiento (SJ, por sus siglas en inglés Squat Jump), de sprint, de repetición máxima (RM) o de dinamometría isocinética e isoinercial (Balsalobre-Fernández, 2015; Halson, 2014).

Y es que, dichos progresos no se deben de quedar en el laboratorio, sino que los entrenadores se deben aprovechar de ello para hacer de su programa de entrenamiento, un proceso mucho más válido y eficiente.

Como se puede observar, y, en conclusión, a la hora de escoger los sistemas de monitorización y cuantificación de la carga de entrenamiento, dado que no existe un método absoluto de referencia que sea aplicable a todos los deportes de resistencia en todas las circunstancias, factores como la accesibilidad, validez, fiabilidad, rentabilidad, esfuerzo, etc., deben ser considerados a la hora de escoger un sistema. Pues todos ellos presentan ventajas e inconvenientes, grados variables de precisión e 
idoneidad en función de la modalidad y la situación de entrenamiento y competición (Mujika, 2017).

En vista de todo lo anterior, el objetivo de esta investigación fue el de ofrecer y aplicar un método de monitorización y cuantificación de la carga, considerando el tipo de atletas, modalidad deportiva y medios al alcance, teniendo en cuenta todas sus variables (frecuencia, volumen e intensidad), e incluyendo tanto aspectos objetivos como subjetivos, así como indicadores externos e internos al deportista, sin entorpecer el proceso del entrenamiento ni ser invasivo para éste.

De este modo, la hipótesis planteada fue que, la carga externa (volumen de kilómetros, intensidad de éstos por velocidad de carrera a partir de unas zonas de entrenamiento determinadas por el perfil individual de lactato y frecuencia de trabajo de los diferentes contenidos) tiene correlación con la carga interna (esfuerzo percibido) $y$ todos ellos con la altura del CMJ, y, por consiguiente, con la fatiga neuromuscular.

\section{MÉTODO}

\section{[Texto borrado]}

\subsection{Muestra}

La muestra de investigación está formada por 5 corredores ( 3 hombres y 2 mujeres) de fondo y medio fondo, con una edad comprendida entre 19 y 24 años

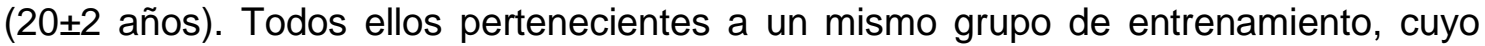
lugar habitual de trabajo es el Centro de Alto Rendimiento (CAR) de Madrid, perteneciente al Consejo Superior de Deportes (CSD).

Respecto a dicha muestra se debe subrayar que, todos ellos han participado en campeonatos nacionales (siendo alguno de ellos incluso campeones y/o medallistas) y han sido seleccionados, en algún momento de su trayectoria, para representar a España en categorías inferiores (pues excepto uno, aún no han alcanzado la categoría absoluta) en diferentes campeonatos internacionales de fondo y medio fondo. De hecho, durante la recogida de datos del presente estudio, varios de ellos son seleccionados para tal fin.

\subsection{Diseño}

El diseño elegido para la elección de la muestra ha sido el de muestreo incidental, en el que no han sido seleccionados al azar, sino por otros motivos como la fácil accesibilidad de los sujetos y su aceptación de querer participar y colaborar en el estudio. Asimismo, de entre todos los atletas que conforman el grupo de prácticas, se escogió a estos 5 deportistas debido a que ellos están becados y, por consiguiente, tienen derecho a una serie de tests de esfuerzo similares que permiten al investigador ajustar mucho más a la hora de conformar sus respectivas zonas de entrenamiento, aspecto clave éste en el desarrollo del estudio.

\subsection{Variables e instrumentos}

Dadas las peculiaridades y condicionantes del lugar de prácticas, y teniendo en cuenta lo que la literatura científica abarca sobre métodos para monitorizar las cargas de entrenamiento, se optó por la propuesta que se expondrá a continuación y que queda resumida y clarificada en la Tabla 2.

Para la frecuencia, se llevó un seguimiento del número de sesiones realizadas y días de descanso, así como de los diferentes tipos de trabajo llevados a cabo. 
En cuanto al volumen, la opción elegida fue la de contabilizar el número de kilómetros por unidad de tiempo. Dicha contabilización, para un mayor detalle, se llevó a cabo manualmente o aprovechando los wearebles, en concreto relojes, que incluían GPS y de los que disponían todos los atletas. Esta tecnología se utilizó, principalmente, en los rodajes, ya que los esfuerzos en la pista de atletismo son relativamente fáciles de hallar sin ello, y, de esta manera, se omite el posible error de medición que te pueden dar.

Respecto al factor intensidad, se utilizaron dos métodos. Uno mediante la clasificación y distribución de los kilómetros recorridos por zonas de entrenamiento. En este caso, se optó por el uso de un indicador de carga externa, como es la velocidad de carrera, pero estableciendo los límites de cada zona a partir de un marcador interno, como es el perfil individual de lactato. Es decir, se individualizó las zonas de entrenamiento de cada atleta en función de la velocidad de carrera que obtuvo a determinadas concentraciones de lactato, extrayendo dichos valores a partir de un test de campo progresivo de lactato y mediante el apoyo de la literatura científica. Las zonas utilizadas fueron:

Primero, a partir del test incremental se pudo obtener:

- Zona 1: < umbral de lactato 1 (LT1), equivalente al umbral aeróbico.

- Zona 2: entre umbral de lactato 1 (LT1) y el umbral de lactato 2 (LT2). Es decir, entre el umbral aeróbico y el anaeróbico.

Estas dos zonas halladas de manera individual (UAI) y no por valores poblacionales, fueron establecidas por uno de los médicos del Consejo Superior de Deportes (CSD). Y es que, está demostrado que, el utilizar como umbral anaeróbico la concentración estándar de lactato en sangre de $4 \mathrm{mmol} / \mathrm{l}$, no es del todo fiable a la hora de representar el máximo estado estable de lactato (MLSS) (Imaz, 2013).

- Zona 3: entre LT2 y la velocidad aeróbica máxima (VAM), estableciendo dicho valor a partir de la velocidad de carrera en la que las concentraciones de lactato de cada individuo superaban los $8 \mathrm{mmol} / \mathrm{l}$, ya que esta concentración (8$9 \mathrm{mmol} / \mathrm{l}$ ) es el referente a nivel poblacional que se suele obtener cuando se alcanza el VO2 máx. (Billat, 2002; Esteve Lanao, 2007; Pallarés y Morán Navarro, 2012). De hecho, en las pruebas de esfuerzo se considera prueba máxima si, desde el punto de vista de la lactacidemia, se superan los $8 \mathrm{mmol} / \mathrm{L}$ (Casajús, Piedrafita y Aragonés, 2009). Dicho límite se halló de esta forma por la imposibilidad de contar con espirometría o cualquier otra prueba de esfuerzo o test de campo.

Sin embargo, más allá de ello, dicho test no ofrecía información, y el establecer como Zona 4 todo lo que esté por encima de la VAM, pareció demasiado amplio, ya que los entrenadores de dicho grupo de atletas suelen diferenciar intensidades más allá de dicho umbral para programar los entrenamientos.

A razón de lo anterior, se decidió establecer otras dos zonas de entrenamiento:

- Zona 4: entre la VAM y la capacidad láctica, estableciendo este límite como el $110 \%$ de la VAM, ya que el rango entre el $105-120 \%$ VAM es el que se suele utilizar para establecer las zonas anaeróbicas lácticas (Billat, 2002). El uso de esta forma para hallar las zonas anaeróbicas es debido a la no existencia de fronteras fisiológicas como en la función aeróbica (Esteve-Lanao, 2007) y a no poder realizar test de campo específicos para ello. 
- Zona 5: > capacidad láctica, es decir por encima del 110\% de la VAM. Corresponde, por consiguiente, a aquellos esfuerzos, sobre todo, de potencia láctica, pues los esfuerzos anaeróbicos alácticos, en las modalidades de resistencia, suelen corresponder con el trabajo de fuerza máxima o explosiva (Cejuela-Anta y Esteve-Lanao, 2011), aspecto éste que se midió mediante el RPE.

La contabilización y clasificación de la distancia recorrida se llevó a cabo manualmente o con los relojes GPS en función del lugar de trabajo. Asimismo, aclarar que, los esfuerzos anaeróbicos alácticos no se encajan en estas zonas de entrenamiento, ya que dichos esfuerzos, en las modalidades de resistencia, suelen corresponder con el trabajo de fuerza máxima o explosiva (Cejuela-Anta y EsteveLanao, 2011), aspecto éste que se medirá, como se verá a continuación, mediante el esfuerzo percibido.

Por otra parte, el otro método utilizado para la variable intensidad, pero desde el punto de vista de la carga interna, fue el del esfuerzo percibido, mediante la Escala de Esfuerzo Percibido (RPE, de sus siglas en inglés Rating Perceived Exertion), y, en concreto, la Escala RPE CR-10. (Borg, 1982; Borg, 1990), cuya numeración para expresar la intensidad va de 0 (reposo) a 10 (máximo) (ver Tabla 1).

Tabla 1. Escala de Esfuerzo Percibido empleada en el estudio. Versión traducida y modificada de la escala RPE CR-10 de Borg (1982; 1990).

\begin{tabular}{cc}
\hline ¿Cómo de intensa te ha parecido la sesión de entrenamiento? \\
\hline 0 & Reposo total \\
\hline 1 & Muy, muy cómoda \\
\hline 2 & Cómoda \\
\hline 3 & Moderada \\
\hline 4 & Algo dura \\
\hline 5 & Dura \\
\hline 6 & - \\
\hline 7 & Muy dura \\
\hline 8 & - \\
\hline 10 & - \\
\hline
\end{tabular}

Nota: RPE CR-10 (Rating Perceived Exertion Category Ratio 10).

Se preguntó al deportista en cada entrenamiento sobre lo intenso que le había parecido éste, siendo 1 "Muy, muy cómodo" y 10 "Máximo" y 0 en caso de reposo total. Asimismo, se pidió al atleta que diferenciara dos valores dentro del mismo entrenamiento, uno haciendo alusión, únicamente, al rodaje y otro a la sesión en general. Todo ello en pos de poder comprobar la carga interna que supone al deportista la realización de los diferentes kilómetros a diferentes intensidades.

$Y$ es que, dicho marcador subjetivo permite valorar aspectos que desde un punto de vista objetivo no es tan viable, como el de la "fatiga mental", definido como un estado psicobiológico causado por periodos prolongados de actividad cognitiva exigente y que se caracteriza por una combinación de manifestaciones subjetivas, conductuales y fisiológicas (Van Cutsem et al., 2017a).

Por último, se midió, semanalmente (los miércoles, antes del inicio de trabajo de la parte principal), la altura en el salto vertical, en concreto, en el CMJ, con el objetivo de comprobar la fatiga neuromuscular acumulada semanalmente que puede generarse en función del volumen total de kilómetros recorridos, la intensidad de éstos según su distribución por zonas de entrenamiento, la frecuencia de trabajo de los 
diferentes tipos de esfuerzos y en relación con los valores medios de RPE. La herramienta utilizada para tal fin fue MyJump, una aplicación de móvil creada por el Dr. Carlos Balsalobre, cuya validación y fiabilidad han sido demostradas en la literatura científica, correlacionando de manera estadísticamente significativa $(r=0.995, P<$ 0.001) los resultados de ésta con los de una plataforma de fuerzas (BalsalobreFernández, Glaister y Lockey, 2015).

Finalmente, también destacar la utilización de la aplicación de móvil whatsapp para contactar con los deportistas en caso de no haber coincidido presencialmente en algún entrenamiento con ellos y, de esta manera, saber cualquiera de las variables citadas (excepto, lógicamente, CMJ, que para su realización es necesario la presencia de ambas partes).

Tabla 2. Resumen de los instrumentos y las variables medidas y registradas en la propuesta de intervención.

\begin{tabular}{|c|c|c|}
\hline \multicolumn{3}{|c|}{ VARIABLES E INSTRUMENTOS DE MEDIDA } \\
\hline Variables medidas & Carga externa & Carga interna \\
\hline & $\begin{array}{c}\text { № de sesiones/unidad de } \\
\text { tiempo. }\end{array}$ & \\
\hline Frecuencia & $\begin{array}{c}\text { № de cada tipo de } \\
\text { contenido/unidad de } \\
\text { tiempo. }\end{array}$ & \\
\hline & $\begin{array}{c}\text { № de kilómetros/unidad de } \\
\text { tiempo. }\end{array}$ & \\
\hline Volumen & $\begin{array}{l}\text { № de kilómetros en cada } \\
\text { zona de } \\
\text { entrenamiento/unidad de } \\
\text { tiempo. }\end{array}$ & \\
\hline & $\begin{array}{c}\text { № de kilómetros de los } \\
\text { rodajes y de las partes } \\
\text { principales/unidad de } \\
\text { tiempo. } \\
\text { (manual y/o relojes GPS) }\end{array}$ & \\
\hline Intensidad & $\begin{array}{l}\text { Zonas de entrenamiento } \\
\text { por velocidad de carrera en } \\
\text { función del perfil individual } \\
\text { de lactato. } \\
\text { (manual y/o relojes GPS) }\end{array}$ & $\begin{array}{l}\text { Esfuerzo percibido } \\
\quad(R P E C R-10)\end{array}$ \\
\hline Fatiga & & $\begin{array}{l}\text { Altura en CMJ } \\
\text { (MyJump) }\end{array}$ \\
\hline
\end{tabular}

${ }^{*}$ Nota: CMJ (Countermovement Jump), GPS (Global Positioning System), RPE CR-10 (Rating Perceived Exertion. Category Ratio-10).

\subsection{Procedimiento}

Para la petición de colaboración de los atletas en la investigación, primero hubo varios diálogos y puestas en común con los entrenadores acerca del procedimiento y objetivos que se persiguen, facilitándoles la información detallada por escrito. Tras su aceptación, se llevó a cabo el mismo procedimiento con los deportistas implicados. 
Los datos se obtuvieron durante 8 semanas, desde el 6 de marzo hasta el 30 de abril (ambos inclusive) del 2017, coincidiendo con el fin de temporada de la pista cubierta y preparación de la pista al aire libre (aunque a varios atletas aún les quedaba alguna competición importante de campos a través). Durante esos casi dos meses, a diario se recogían los datos expuestos anteriormente (excepto el CMJ, que era semanalmente) a los 5 atletas correspondientes.

Luego, también destacar que, aquellas semanas en las que debido a lesión el atleta no podía proceder a realizar el CMJ, lógicamente, en ese apartado estadístico se anularon. También hubo imposibilidad de medir el CMJ en la semana correspondiente a Semana Santa, ya que cada atleta estaba en un distinto punto geográfico de vacaciones y/o concentración.

Por último, también aclarar que, las competiciones desarrolladas durante este periodo, de igual manera, se contabilizaron y registraron como cualquier entrenamiento, ya que no deja de ser una carga (con su correspondiente estrés) más para el organismo. De hecho, no hay entrenamiento más específico que el de la propia competición. De esta manera, también se puede comprobar, si fuera del interés, si durante las semanas que se compite, y principalmente, las de mayor peso, la carga de entrenamiento se ve reducida/modificada (ya sea en volumen, frecuencia $\mathrm{y} / \mathrm{o}$ intensidad) para llegar en óptimas condiciones a la competición, es decir, lo que se conoce como tapering (Mujika, 2010; Mujika et al., 2002).

\subsection{Análisis estadísticos}

Para los estudios estadísticos, en un primer momento, se llevó a cabo un análisis descriptivo, indicando los sumatorios y/o medias de cada atleta respecto a las diferentes variables que conforman la carga de entrenamiento (intensidad, volumen y frecuencia de los diferentes contenidos). Asimismo, para comprobar las relaciones entre los parámetros de carga externa y carga interna, se optó por realizar un análisis de correlaciones bivariadas de Pearson. Dicho análisis de datos se efectuó con el programa IBM SPSS Statistics 24.

\section{RESULTADOS}

\subsection{Análisis descriptivos}

En este primer apartado se expondrán aquellos datos de un mayor carácter descriptivo, es decir, aquella cuantificación que nos revela información sobre el volumen, la frecuencia y la intensidad de los entrenamientos, sin entrar en análisis estadísticos, como si de entrenadores o preparadores físicos de los atletas en cuestión fuésemos.

Así pues, de estas ocho semanas de monitorización, se obtuvieron las siguientes cuantías:

\section{Volumen e intensidad}

Respecto a la variable volumen, como ya se ha argumentado previamente, se contabilizó mediante el número de kilómetros, diferenciando, asimismo, entre los efectuados en los rodajes y los llevados a cabo en las partes principales de cada sesión (ver Tabla 3). 
Asimismo, y ya adentrándonos también en la variable intensidad, se diferenció la cuantía de kilómetros efectuados en cada zona de entrenamiento específica a cada uno de los atletas en función de la velocidad de carrera según su perfil individual de lactato, tanto de los rodajes y de las partes principales por separado (ver Tabla 3), como de forma conjunta (ver Tabla 4).

Tabla 3. Volumen de kilómetros e intensidad de éstos por zonas de entrenamiento, diferenciando entre los rodajes y la parte principal, durante las 8 semanas de recogidas de datos, de cada uno de los atletas.

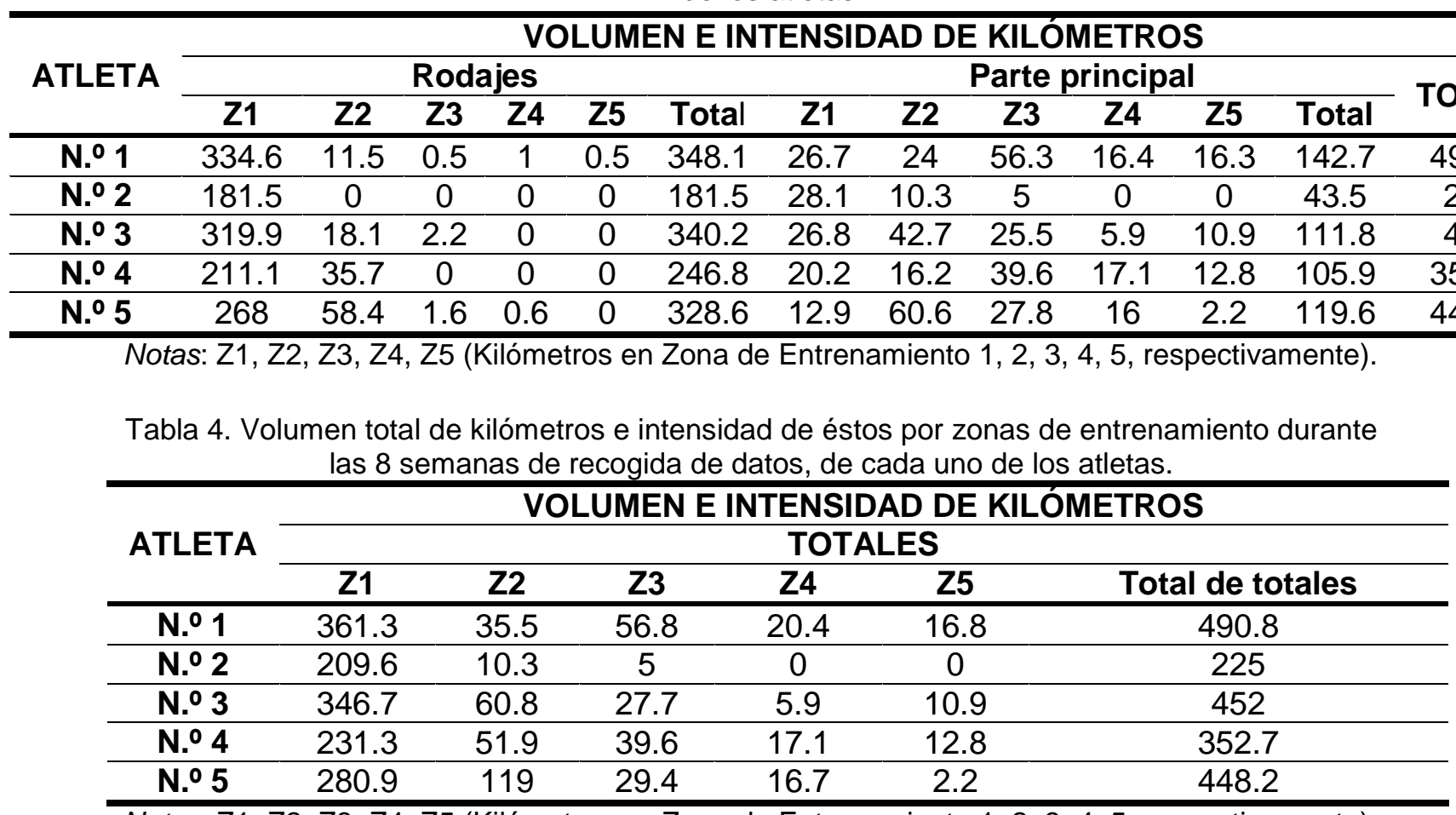

Notas: Z1, Z2, Z3, Z4, Z5 (Kilómetros en Zona de Entrenamiento 1, 2, 3, 4, 5, respectivamente).

En la Figura 1 se puede apreciar, de manera visual, el volumen de kilómetros en cada zona de entrenamiento de cada atleta durante toda la investigación.

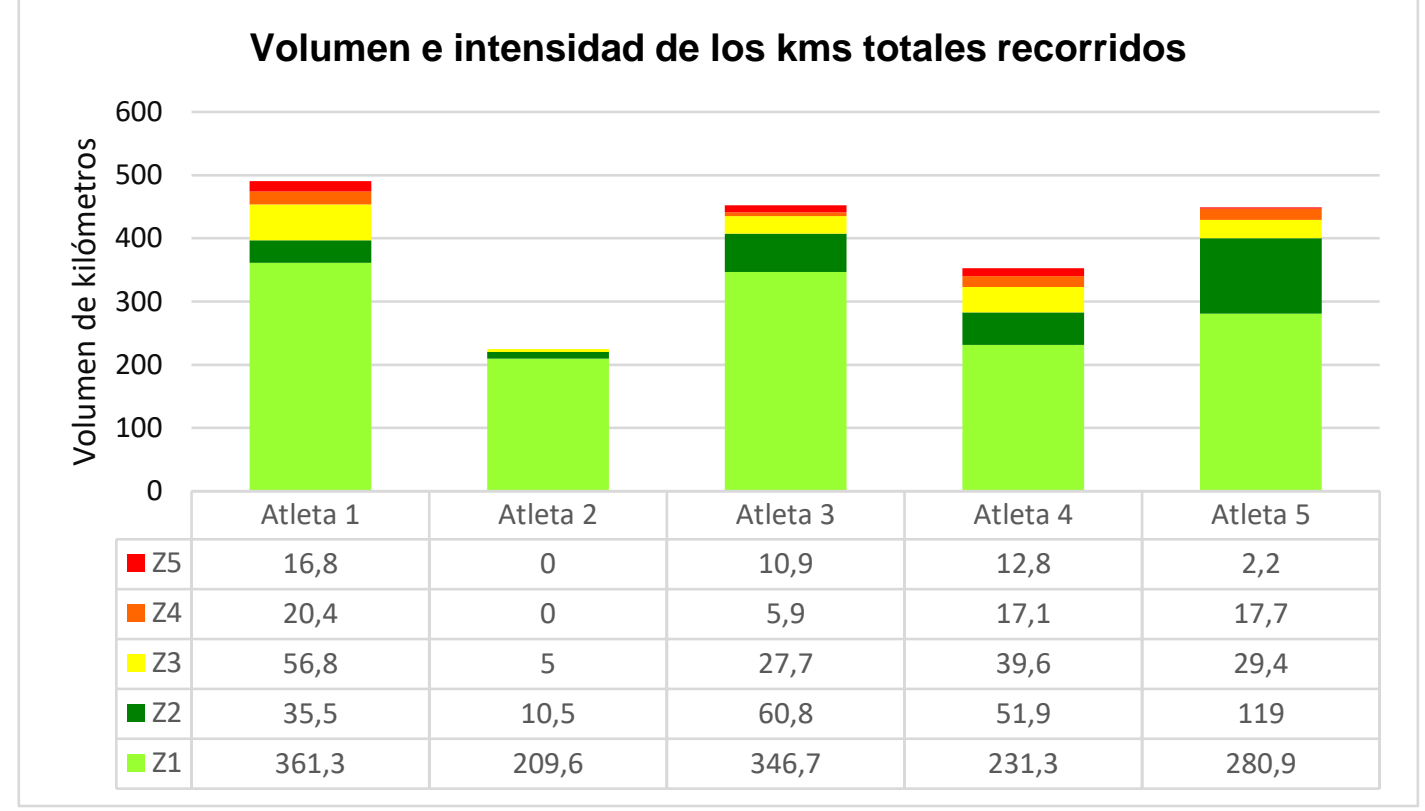

Nota: Z1, Z2, Z3, Z4 y Z5 (Zona de entrenamiento 1, 2, 3, 4 y 5, respectivamente). 
Figura 1. Volumen de kms (kilómetros) totales de cada uno de los 5 atletas durante las 8 semanas de recogida de datos, distribuidos por intensidad (zonas de entrenamiento).

Asimismo, si se hace un sumatorio, entre los 5 deportistas, del total de kilómetros recorridos en cada zona de entrenamiento, se puede apreciar las diferencias de volumen a cada intensidad que han llevado a cabo durante estos dos meses (ver Figura 2).

\section{Kilómetros totales distribuidos entre las 5 zonas de entrenamiento.}

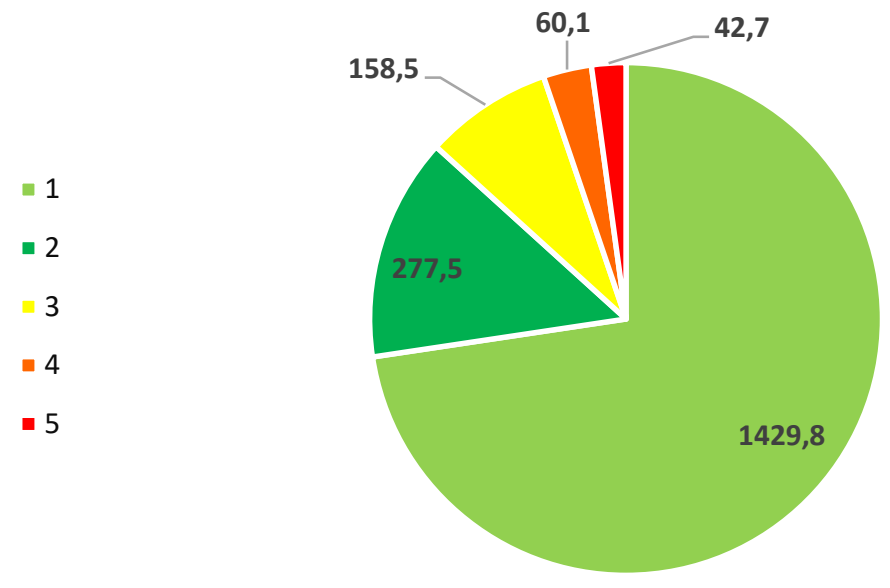

Nota: 1, 2, 3, 4 y 5 corresponden a la numeración de las diferentes zonas de entrenamiento. Figura 2. Kilómetros totales recorridos entre los 5 atletas durante las 8 semanas de recogida de datos y distribuidos por zonas de entrenamiento.

\section{Frecuencia}

Por otro lado, respecto a la otra gran variable estudiada en relación a la carga de entrenamiento, es decir, la frecuencia, se contabilizaron tanto el número total de sesiones de entrenamiento, como la media que ha supuesto en estas 8 semanas de trabajo. De igual manera, y al ser la otra cara de la moneda, se llevó a cabo el mismo procedimiento con los días de "Descanso total" (ver Tabla 5).

Tabla 5. Número total y media a la semana de frecuencia de sesiones de entrenamiento durante las 8 semanas de recogida datos de cada uno de los atletas.

\begin{tabular}{|c|c|c|c|c|}
\hline \multirow{3}{*}{ ATLETA } & \multicolumn{4}{|c|}{ FRECUENCIA DE SESIONES DE ENTRENAMIENTO } \\
\hline & \multicolumn{2}{|c|}{ SESIONES DE TRABAJO } & \multicolumn{2}{|c|}{ DESCANSOS TOTALES } \\
\hline & N. TOTAL & MEDIA & N.ㅇ TOTAL & MEDIA \\
\hline N.․ 1 & 48 & 6 & 8 & 1 \\
\hline NN. $=2$ & 39 & 4.88 & 17 & 2.13 \\
\hline N.. 3 & 49 & 6.13 & 7 & 0.88 \\
\hline N. 04 & 38 & 4.75 & 18 & 2.25 \\
\hline N. 5 & 39 & 4.88 & 17 & 2.13 \\
\hline
\end{tabular}

Nota: en dicho recuento se incluyen las competiciones, pues se las trata como el tipo de entrenamiento más específico posible.

Finalmente, la principal peculiaridad de este estudio fue la de diferenciar los contenidos trabajados, cuantificando, de esta manera, los diferentes tipos de esfuerzos llevados a cabo durante el periodo de registro, tanto en niveles absolutos como relativos (ver Tabla 6). 
Tabla 6. Número total y media a la semana de frecuencia de trabajo de los principales contenidos durante las 8 semanas de recogidas de datos de cada uno de los atletas.

\begin{tabular}{|c|c|c|c|c|c|c|c|c|c|c|c|c|c|c|c|c|c|c|c|c|c|c|c|c|}
\hline \multirow{3}{*}{ AT } & \multicolumn{24}{|c|}{ FRECUENCIA DE CONTENIDOS } \\
\hline & \multicolumn{2}{|c|}{ Rodajes } & \multicolumn{2}{|c|}{ Control. } & \multicolumn{2}{|c|}{ C.R. } & \multicolumn{2}{|c|}{ Series } & \multicolumn{2}{|c|}{ Cuestas } & \multicolumn{2}{|c|}{ Fuerza } & \multicolumn{2}{|c|}{ Core } & \multicolumn{2}{|c|}{ Elíptica } & \multicolumn{2}{|c|}{ Bici } & \multicolumn{2}{|c|}{ P.O. } & \multicolumn{2}{|c|}{ Competic. } & \multicolumn{2}{|c|}{ Liebre } \\
\hline & $\mathbf{T}$ & M & $\mathbf{T}$ & M & $\mathbf{T}$ & M & $\mathbf{T}$ & $\mathbf{M}$ & $\mathbf{T}$ & $\mathbf{M}$ & $\mathbf{T}$ & $\mathbf{M}$ & $\mathbf{T}$ & $\mathbf{M}$ & $\mathbf{T}$ & $\mathbf{M}$ & $\mathbf{T}$ & $\mathbf{M}$ & $\mathbf{T}$ & $\mathbf{M}$ & $\mathbf{T}$ & M & $\mathbf{T}$ & M \\
\hline $\begin{array}{c}\text { N.o } \\
1\end{array}$ & 48 & 6 & 8 & 1 & 4 & 0.5 & 12 & 1.5 & 6 & 0.8 & 13 & 1.6 & 15 & 1.9 & 0 & 0 & 0 & 0 & 1 & 0.1 & 1 & 0.1 & 1 & 0.1 \\
\hline $\begin{array}{c}\text { N.o } \\
2\end{array}$ & 25 & 3.1 & 0 & 0 & 3 & 0.4 & 4 & 0.5 & 0 & 0 & 14 & 1.8 & 22 & 2.8 & 0 & 0 & 6 & 0.8 & 0 & 0 & 1 & 0.1 & 0 & 0 \\
\hline $\begin{array}{c}\text { N.o } \\
3\end{array}$ & 42 & 5.3 & 4 & 0.5 & 3 & 0.4 & 8 & 1 & 2 & 0.3 & 11 & 1.4 & 16 & 2 & 15 & 1.9 & 2 & 0.3 & 1 & 0.1 & 0 & 0 & 0 & 0 \\
\hline $\begin{array}{c}\text { N.o } \\
4\end{array}$ & 33 & 4.1 & 4 & 0.5 & 5 & 0.6 & 7 & 0.9 & 5 & 0.6 & 7 & 0.9 & 10 & 1.3 & 0 & 0 & 0 & 0 & 1 & 0.1 & 3 & 0.4 & 0 & 0 \\
\hline $\begin{array}{c}\text { N.o } \\
5\end{array}$ & 38 & 4.8 & 3 & 0.4 & 2 & 0.3 & 10 & 1.3 & 1 & 0.1 & 1 & 0.1 & 4 & 0.5 & 0 & 0 & 1 & 0.1 & 0 & 0 & 4 & 0.5 & 0 & 0 \\
\hline
\end{tabular}

Nota: "AT" (Atleta); "T" (N. ${ }^{\circ}$ Total); "M" (Media, "T"/8); "Control." (Controlados); "C. R." (Cambios de ritmo); "P. O." (Preparación de obstáculos); "Competic." (Competiciones); "Liebre" (Hacer de liebre en alguna competición).

\subsection{Análisis de correlaciones}

En cuanto a la variable de carga interna "Altura en el CMJ", al realizar una correlación con las variables de carga externa del volumen (número de kilómetros) y la intensidad (zonas de entrenamiento por velocidad de carrera a partir del perfil de lactato y umbrales individuales), se pudo comprobar que, existe una relación inversa entre sí (tanto con el cómputo total de kilómetros, como con las diferentes zonas), aunque siendo, solamente, significativa $(p<.05)$ en Z3 $(-.892)$ y Z4 $(-.937)$.

Por otro lado, se correlacionó la altura del CMJ con la otra gran variable de carga externa estudiada, es decir, la frecuencia de los diferentes contenidos de trabajo. En dicho análisis se puede comprobar la relación negativa y significativa $(r=-$ .960 con una $p<.01$ ) entre la altura del CMJ y el número de series realizadas a la semana. Del resto de contenidos trabajados no existe correlación significativa, pero, sin embargo, se puede comprobar como con trabajos que se pueden considerar más intensos (controlados o cuestas), la correlación es negativa $(r=-.847$ y $r=-.669$, respectivamente), mientras que con otros que, a priori, suelen ser menos intensos en estos deportistas (por ejemplo, bicicleta o core) la correlación es positiva ( $r=.850$ y $r=$ 622 , respectivamente).

Una vez analizada la altura en CMJ con todas las variables de carga externa estudiadas, se optó por llevar a cabo el mismo procedimiento con la otra variable de carga interna registrada, dícese la Escala de Esfuerzo Percibido.

Primero se correlacionó el RPE de cada semana con el volumen de kilómetros totales y con el volumen de kilómetros recorrido en cada zona de entrenamiento en dicha franja temporal. Los resultados nos muestran, por un lado, que existe una relación positiva significativa $(r=.734$ con una $p<.01)$ entre el volumen de kilómetros totales y el RPE semanal, y, por otro lado, que hay una relación también positiva entre dicha variable subjetiva y los kilómetros recorridos en cada zona de entrenamiento, pero con diferente significación: Z1, Z3 y Z4 $(p<.01)$, Z5 $(p<.05)$ y Z2 (sin significatividad) (ver Tabla 7). 
Tabla 7. Correlaciones entre el RPE semanal y el volumen e intensidad de la carga en dicho periodo.

\begin{tabular}{|c|c|c|c|c|c|c|c|}
\hline \multicolumn{8}{|c|}{ Correlaciones RPE con volumen e intensidad de la carga a la semana } \\
\hline & $\begin{array}{c}\mathrm{Kms} \\
\text { totales }\end{array}$ & Kms Z1 & Kms Z2 & Kms Z3 & Kms Z4 & Kms Z5 & RPE \\
\hline $\begin{array}{c}\mathrm{Kms} \\
\text { totales }\end{array}$ & & .927 & .412 & .563 & .345 & .354 & $.734^{\star \star}$ \\
\hline Kms Z1 & & & .294 & .423 & .165 & .291 & $.577^{\star \star}$ \\
\hline Kms Z2 & & & & .019 & .144 & .119 & .292 \\
\hline Kms Z3 & & & & & 339 & .494 & $.559^{\star \star}$ \\
\hline Kms Z4 & & & & & & .157 & $.528^{\star \star}$ \\
\hline Kms Z5 & & & & & & & $.375^{\star}$ \\
\hline RPE & & & & & & & \\
\hline
\end{tabular}

Nota: ${ }^{*} p<.05 ;{ }^{* *} p<.01$; Kms (Kilómetros); Z1, Z2, Z3, Z4, Z5 (Zona de Entrenamiento 1, 2, 3, 4, 5, respectivamente); RPE (Rating Perceived Exertion).

Posteriormente, se decidió realizar el mismo análisis por totales (sumatorio de kilómetros en cada zona de entrenamiento de los 2 meses analizados), para comprobar el peso de cada intensidad en el RPE total (media de las 8 semanas). En este caso, las correlaciones, a pesar de no ser significativas, siguieron siendo, lógicamente, positivas, y se pudo observar cómo a mayor intensidad, mayores suelen ser los valores en RPE, excepto en Z5, probablemente como consecuencia de las variables extrañas que han ido surgiendo a lo largo de este estudio y que se comentarán en el apartado de discusión, las cuales han hecho que el volumen de kilómetros recorridos a dichas intensidades, sea muy escaso (ver Figura 2), habiendo muchas semanas en las que los atletas no recorrían ni un metro por encima de la capacidad láctica (ver Figura 3).

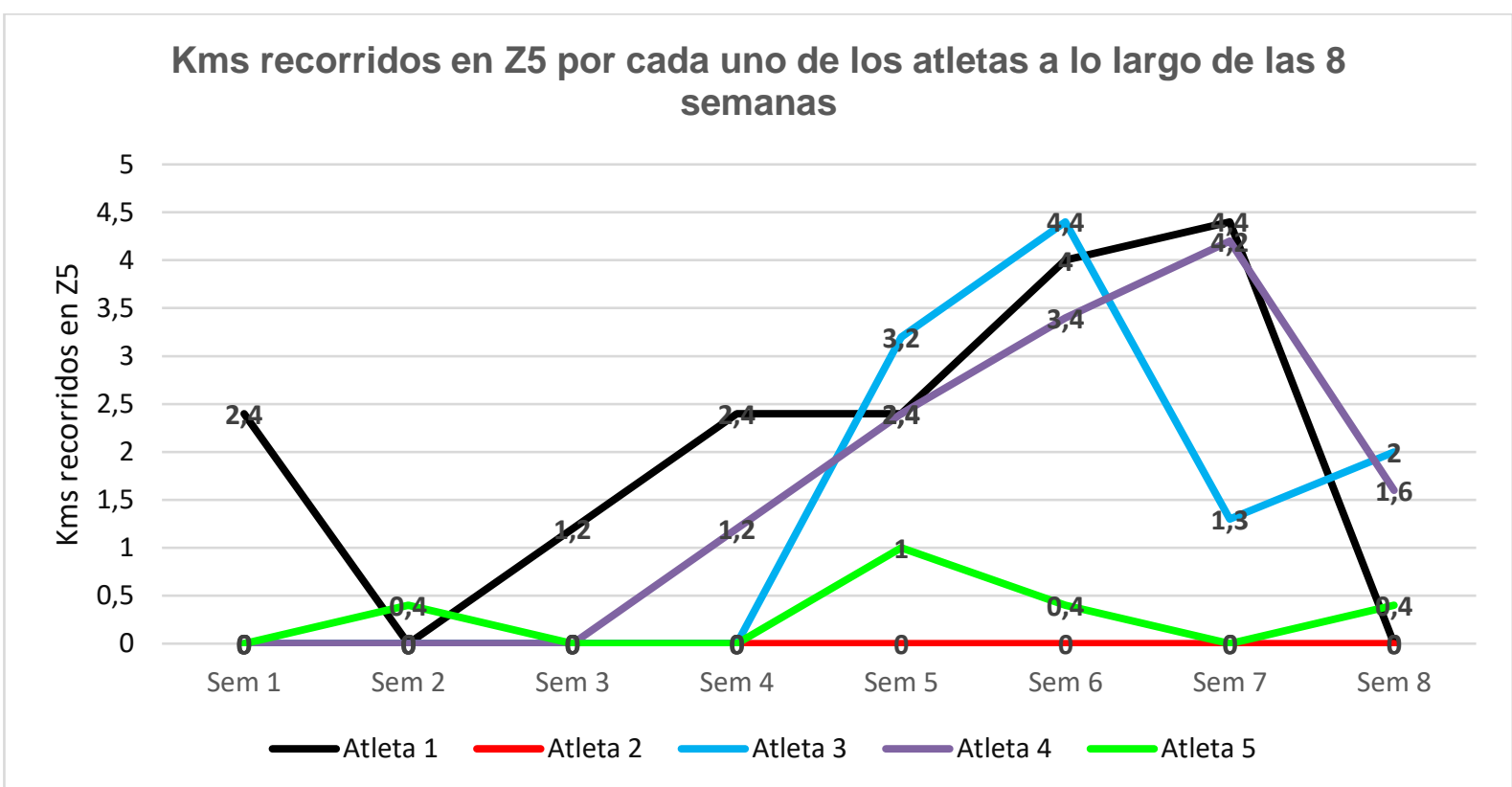

Figura 3. Kilómetros recorridos en Z5 (Zona de entrenamiento 5) a lo largo de las 8 semanas por cada uno de los 5 atletas.

En segundo lugar, se correlacionó el RPE con la frecuencia de los diferentes tipos de contenidos de trabajo a la semana. En dicho análisis, sólo salió significativo ( $p$ $<$.05) la relación inversa entre el RPE y el trabajo de bicicleta. No obstante, también se puede apreciar la relación (aunque no significativa), también inversa, como se 
podría esperar, con otros tipos de trabajo más livianos, como el core, o los descansos totales, y la relación directa con la mayoría del resto de tipologías.

Por último, lo que se planteó fue comprobar la relación que podría haber entre las dos variables de carga interna, es decir, el RPE con la altura en el CMJ. La relación salió inversa, como podría esperarse, pero sin significación.

\section{DISCUSIÓN}

Este trabajo pretendió sugerir la importancia de llevar a cabo una correcta monitorización y cuantificación de las cargas de entrenamiento en la práctica deportiva, y más concretamente, en las modalidades de fondo y medio fondo de carrera con atletas de alto nivel.

Asimismo, además de aplicar un sistema de seguimiento de cada una de las variables principales que conforman la carga de trabajo, se ha perseguido relacionar la carga externa con la interna, en pos de poder establecer correlaciones que enriquezcan y detallen la información de este proceso.

Dicho lo cual, se desglosará la discusión, de manera detallada, siguiendo el objetivo e hipótesis citados con anterioridad, empezando por esta última.

La hipótesis mencionaba, "La carga externa (volumen de kilómetros, intensidad de éstos por velocidad de carrera a partir de unas zonas de entrenamiento determinadas por el perfil individual de lactato y frecuencia de trabajo de los diferentes contenidos) tiene correlación con la carga interna (esfuerzo percibido) y todos ellos con la altura del CMJ, y, por consiguiente, con la fatiga neuromuscular". La hipótesis se cumple, ya que los marcadores internos del RPE y altura en el CMJ han demostrado relación con los indicadores externos, aunque algunas no han sido significativas, como se verá a continuación.

Llevando un orden paralelo al establecido en el apartado Análisis correlacional, la primera conexión que se comprueba es entre la altura del CMJ y el volumen total y por zonas de entrenamiento (intensidad). En ésta se puede apreciar la relación inversa, entre el número de kilómetros realizados la semana previa al CMJ y la altura en dicho salto.

Es decir, a priori (pues además no es significativa la relación), por una parte, a más volumen total de kilómetros a la semana, menos altura en el CMJ se consigue, tal y como ya habían demostrado estudios como el de Balsalobre-Fernández, TejeroGonzález y del Campo-Vecino (2014).

Por otra parte, a mayor acumulación de kilómetros en cada zona de entrenamiento, también menor es el rendimiento que se consigue en dicho salto vertical. Ahora bien, respecto a la intensidad, y he aquí lo interesante respecto a dicha variable, las relaciones son significativas en Z3 y Z4, por lo que se puede deducir que, no sólo depende la distancia que se acumule, sino también la intensidad a la que se recorra ésta, pues tal y como nos argumentan Tuimil, Iglesias, Dopico y Morenillas (2005), a mayor participación del componente anaeróbico, mayor (o al menos diferente) es el estrés interno.

Sin embargo, hay otros estudios como el ya citado de Balsalobre-Fernández et al. (2014), que establecen una relación positiva y significativa entre la zona de entrenamiento y la altura en el CMJ. Esto puede ser debido por una simple razón, y es 
que, en contraposición, el volumen de kilómetros que se hacen a intensidades cada vez mayores es menor. A razón de ello, es probable que ésta sea la razón por la que, en nuestro estudio, en la zona 5, la correlación no llegue a ser significativa, pues el volumen de kilómetros que se hace por encima de la capacidad anaeróbica láctica, es decir, aquellos esfuerzos correspondientes a la potencia anaeróbica láctica son escasos.

En resumen, en un principio, a mayor número de kilómetros, mayor es el estrés interno que se genera en el atleta y, por lo tanto, menor el rendimiento en el CMJ. Asimismo, comparando cada zona de entrenamiento de forma aislada, a mayor número de kilómetros en cada una de ellas, lógicamente, el resultado será el mismo que el anterior. Sin embargo, si se analiza la intensidad sin tener en cuenta el volumen de kilómetros realizados, la relación con el CMJ puede resultar positiva, ya que, a mayor intensidad, menos volumen de kilómetros se suelen llevar a cabo.

Probablemente, sea por ello que, en el estudio, las zonas intermedias-altas (Z3 y Z4) son las que salen significativamente estadísticas, ya que si se mide la relación volumen-intensidad, de forma conjunta, en el rendimiento del CMJ, son las zonas en las que la relación intensidad-volumen es la más equilibrada, en el sentido de que, por debajo de dichas zonas, el volumen es más alto, pero la intensidad es bastante escasa (por debajo del umbral anaeróbico), y en la zona 5, la intensidad es algo más alta (por encima de la capacidad láctica) pero el volumen que se lleva a cabo en dicha zona, en la muestra seleccionada, es muy escueto en comparación a las demás.

Dicho lo cual, en relación a lo anterior, un método que, a lo mejor, hubiera permitido ver, de manera más fidedigna, la relación entre la intensidad y el volumen (de manera conjunta) con la altura en el CMJ, es la variante del RPE denominada session RPE, creada por Foster (1998) y defendida, posteriormente, por otra gran cantidad de estudios en deportes de resistencia, como el de Manzi et al. (2015) o el de Mujika (2017). Este método consiste en valorar la carga general de una sesión, multiplicando la puntuación que otorga el deportista al entrenamiento (RPE CR-10) por la duración de éste, es decir, intensidad por volumen. Sin embargo, dicho método no se eligió debido a la necesidad de tener que cuantificar el volumen mediante el tiempo, en lugar de distancia recorrida, y, sobre todo, debido al variable y relativo gran espacio de tiempo que se interponía entre el rodaje y la parte principal de la sesión en numerosas ocasiones, lo que se creyó que podía interferir en la percepción del esfuerzo, pues la variable densidad no la tiene en cuenta, siendo éste uno de sus principales inconvenientes (Cejuela-Anta y Esteve-Lanao, 2011).

En definitiva, en vistas de estos resultados, y tal y como nos recalcan estudios como el de Mujika (2010), la clave a la hora de conseguir un mayor rendimiento en las semanas de tapering podría no estar, tanto, en reducir la intensidad, sino en reducir el volumen a dichas intensidades, que no es lo mismo.

Seguidamente, el otro análisis correlacional que se llevó a cabo fue el de la altura del CMJ con la frecuencia de los diferentes contenidos de trabajo. Sobre esto, lo más parecido que se ha podido encontrar en la literatura científica son artículos que comparan las diferencias en cuanto a frecuencia de sesiones de trabajo entre semanas en las que se busca el afinamiento del deportista para que alcance el máximo rendimiento en una determinada competición objetivo. Así, por ejemplo, el trabajo de Mujika et al. (2002) demostró que, contra pronóstico, el mantener una sesión de trabajo al día (sesión diaria de HIT, pero con reducción progresiva del volumen), en lugar de reducir dicha variable un 33\% (descanso cada 3 días) los 6 días previos de tapering, hizo que los atletas de medio fondo de carrera obtuvieran mejor rendimiento. Ello nos vuelve a recalcar la importancia de, a la hora de conseguir un mayor afinamiento en nuestros atletas, no hacer tanto hincapié en la reducción de la 
frecuencia, sino en el volumen, tal y como pasaba al relacionarlo con la variable intensidad.

Ahora bien, el presente estudio no es del todo similar, pues el rendimiento no se equipará con la distancia de carrera, sino con el indicador de fatiga neuromuscular que te puede ofrecer la altura del salto vertical (CMJ) contrastándolo de manera longitudinal. Además, se intentó ir un paso más allá, y no sólo se diferenció entre días de descanso y días de trabajo, sino también, se distinguió, dentro de los días de entrenamiento, el tipo de contenido que se había llevado a cabo, asumiendo que, no sólo influye las variables de volumen, intensidad y la misma frecuencia, sino también la naturaleza de lo trabajado.

Así pues, las correlaciones al respecto muestran, únicamente, una relación significativa y negativa, que es con la frecuencia de trabajo a la semana de series. En vistas a estos resultados, la lectura más razonable es la de que, cuando se trabaja series, dichos esfuerzos consisten en HITs, con intensidades que rondan a la PAM (potencia aeróbica máxima) y provocando, por consiguiente, que la fatiga tanto muscular como neural, y en definitiva el estrés interno, se vea incrementado (Fernández-del Olmo et al., 2013; Tuimil et al., 2005).

Sin embargo, si bien es verdad que lo anterior tiene toda su lógica y está corroborado, si lo relacionamos con las conclusiones de la anterior correlación (volumen e intensidad) nos daremos cuenta de que dicha conclusión, cuanto menos, hay que tomarla con cautela. A lo que se quiere llegar es que, al igual que no sólo hay que fijarse en la intensidad a la hora de sacar conclusiones para el rendimiento en el CMJ, pues el volumen afecta en gran medida, en el caso de la frecuencia ocurre de igual manera. Por ejemplo, no es lo mismo, y, por ende, seguramente, no provoque una misma fatiga neuromuscular, unas series de $5 \times 300$ metros al $120 \%$ de la PAM, que $10 \times 300$ metros al $100 \%$ de la PAM.

Respecto al resto de contenidos, como se ha dicho anteriormente, no se han encontrado relaciones significativas, aunque se puede apreciar la relación inversa entre la altura del CMJ con los controlados, los rodajes y las cuestas, y, positiva, con el core y bicicleta. El primer grupo, seguramente, por sus requerimientos relativamente altos en volumen de trabajo y/o intensidad, además de que la pendiente de las cuestas provoca una añadida demanda muscular. Los segundos, probablemente, al mínimo estrés interno que generan. El core debido a su naturaleza y forma de trabajarlo que tenían los entrenadores y el trabajo de bicicleta porque únicamente se llevaba a cabo como alternativa en caso de lesión. Sin embargo, no se han encontrado investigaciones al respecto, y se deberían hacer más estudios para poder sacar alguna conclusión en firme. Además de que, al igual que pasa con las series, las demandas dentro de una misma categoría pueden variar.

Por último, a destacar respecto a la frecuencia, es la diferenciación entre el número de días de descanso total y los días de trabajo (sea cual sea el contenido realizado) y la influencia que puede tener ello en la altura del CMJ. Los resultados de la correlación muestran una relación positiva en el caso de los descansos y negativa con los días de entrenamiento, aunque ambas correlaciones son muy débiles.

Ello se pueda deber, primero, y nuevamente, a que la variedad de contenidos, volumen e intensidad de las diferentes sesiones es un claro factor a tener en cuenta sobre el tipo y cantidad de estrés interno que se provoca en el atleta, y segundo, y tal y como ya se ha citado en el trabajo de Mujika et al. (2002), el aumento en la frecuencia de descansos (o reducción de entrenamientos) no tiene por qué tener una correlación inversa significativa con el aumento del rendimiento. 
Una vez comentadas todas las relaciones entre el CMJ y los diferentes indicadores de carga externa observados, se pasará a continuación a realizar el mismo procedimiento con el RPE.

Respecto al volumen total de kilómetros a la semana, éste expresa una asociación con el RPE positiva y significativa, lo que significa que, a mayor número de kilómetros que se recorran a la semana, mayor será el esfuerzo percibido por el atleta, tal y como se podría esperar y cómo ya habían demostrado otras investigaciones como la de Balsalobre-Fernández et al. (2014).

De igual manera, en cuanto a la relación del RPE con el volumen de kilómetros recorridos a la semana en cada una de las 5 zonas de entrenamiento, todas ellas muestran una relación positiva y significativa, excepto en Z2 que sólo cumple la primera de las premisas. Así pues, de entre las significaciones, Z1, Z3 y Z4 muestran la relación más fuerte, seguido de Z5.

Dichos resultados, grosso modo, son los esperados, pues como ocurría con el volumen total de kilómetros a la semana (sin establecer zonas), lo normal es que, cuanta más distancia se recorra por unidad de tiempo, mayor sea el esfuerzo percibido en el deportista.

El que se produzcan diferentes grados de significación entre las diferentes intensidades (zonas de entrenamiento), puede que sea debido, tal y como ya ocurría con el CMJ, a que la intensidad, es este estudio, se haya relacionado unida con el volumen. Es decir, el que Z1 tenga tanta significación a pesar de representar las intensidades más bajas, puede ser debido al alto volumen de kilómetros que se recorren en esa zona (la más cuantiosa con diferencia). Y respecto a Z3 y Z4, seguramente, debido a la relación equilibrada entre kilómetros recorridos e intensidad de éstos (ver Figura 2).

De esta manera, Z5 tiene una relación significativa, pero más baja, a pesar de plasmar las intensidades más altas, probablemente, como consecuencia del poco volumen que se ha llevado a cabo a esos ritmos de carrera, pues, además, esos dos meses correspondieron al fin de la temporada de pista cubierta e inicio de la pista al aire libre (con lo que ello conlleva, que entre otras cosas supone la no realización de sesiones extremadamente duras). Asimismo, coincidió con Semana Santa, días de descanso para algunos atletas como consecuencia del fin de temporada de la pista cubierta y varias lesiones que imposibilitaron seguir la programación esperada (ver Figura 1 y Figura 3). De igual manera, la similitud de esfuerzos percibidos en los trabajos anaeróbicos lácticos, sin haber diferencias notarias entre el trabajo de capacidad y potencia, puede que también haya podido influir.

Por último, la no significación de Z2 tendría una explicación similar, y es que, si cierto es que la intensidad respecto a Z1 es algo superior, en contraposición, el volumen de kilómetros que se han recorrido en esa zona es muy inferior, tanto a nivel general (ver Figura 2) como de manera particular en cada uno de los atletas (ver Figura 1).

Además, si analizamos los límites/umbrales que dividen cada zona de entrenamiento, veremos que, entre $Z 1$ y Z2 se sitúa el umbral aeróbico, cuya superación no supone una fatiga añadida excesiva en los atletas, y, probablemente, en menor medida, si éstos son de resistencia, pues están acostumbrados a realizar esfuerzos de larga duración. Sin embargo, entre Z2 y Z3 y entre Z3 y Z4 se encuentran el umbral anaeróbico y la VAM, respectivamente, cuyo traspaso sí que supone un mayor estrés fisiológico en el deportista. 
Se debe destacar la influencia que tiene en la fatiga no sólo la intensidad, sino también su relación con el volumen. Al respecto, el concepto ya citado de "fatiga mental" coge relevancia. Van Cutsem et al. (2017a), en la revisión bibliográfica que llevaron a cabo, comprobaron que la principal consecuencia de este estado es una disminución del rendimiento, asociada con una percepción del esfuerzo más alta de lo normal. Ello puede hacer comprender, nuevamente, la importancia de medir el carácter subjetivo del esfuerzo en los atletas, y, por consiguiente, su fatiga mental, sobre todo, si la modalidad deportiva de éstos conlleva esfuerzos prolongados en el tiempo.

Seguidamente, se decidió realizar esta misma comparativa no por semanas, sino por totales (sumatorio de las 8 semanas), es decir, comparando la media del RPE de las 8 semanas con el sumatorio de kilómetros recorridos en dicho periodo de tiempo. En este caso, las correlaciones no llegan a ser significativas, pero todas ellas son positivas y cada vez más fuertes a medida que la intensidad (zona de entrenamiento) es mayor (excepto en Z5, probablemente como consecuencia de lo explicado en el anterior párrafo). Son numerosas las investigaciones que corroboran cómo a mayor estrés interno, mayor es el esfuerzo percibido, como la de Lambert y Borresen, 2010 o la de Wallace et al., 2014, y que, por consiguiente, éste, en cualquiera de sus variedades, puede ser un indicador fiable para valorar la carga interna (Pageaux, 2016).

Asimismo, el que ninguna de estas últimas correlaciones salga significativa, probablemente se pueda deber a las comentadas ya variables extrañas (lesiones, vacaciones, días de descanso, etc.) que no han podido ser controladas y que, dadas las limitaciones del estudio, como la escasa muestra y el corto periodo de tiempo de la investigación, el peso e influencia de éstas en los resultados finales sea bastante grande.

Por otro lado, al relacionar el RPE con la frecuencia de los diferentes contenidos de trabajo, el análisis correlacional nos muestra una única relación significativa, la cual, además, es positiva, que es con el trabajo de bicicleta. Todo ello hace pensar que, como ya ocurría al relacionar dicha variable de entrenamiento con el CMJ, si bien es verdad que hay ciertos contenidos que suelen generar mayor estrés interno y, por consiguiente, mayor esfuerzo percibido que otros, las diferencias de volumen e intensidad dentro de un mismo tipo de contenido, hace que el valor que se le asigne de RPE a cada contenido pueda diferir en gran medida de un día a otro.

Una posible solución sería comparar el esfuerzo percibido por separado para cada tipo de contenido, y no de manera conjunta, con el fin de poder establecer diferencias y comparaciones dentro de un mismo tipo de esfuerzo. Ya que, por ejemplo, habrá semanas donde el trabajo de fuerza tenga un RPE inferior en el atleta que, con el trabajo de cuestas, y en otras ocasiones sea al revés, sobre todo (aunque no únicamente), como consecuencia del volumen e intensidad de cada sesión, por lo que no se podrá afirmar que un tipo de trabajo u otro provoca mayor estrés interno. Sin embargo, si relacionas dos sesiones de una misma naturaleza, por ejemplo, las cuestas, si un día el RPE es mayor que otro, sí que se podrá estudiar, los porqués de ello, como el número y/o distancia de las series, la pendiente, los descansos, etc.

Finalmente, una vez analizadas las correlaciones entre la altura del CMJ con todas las variables de carga externa medidas y haber hecho el mismo procedimiento con el RPE, el siguiente y último análisis fue el de comprobar la relación entre esos dos mismos marcadores, es decir, entre la altura en el salto vertical con contramovimiento y el esfuerzo percibido. 
En el caso de este estudio, la relación entre ambos parámetros fue inversa, como se cabía esperar, y tal y como abogan investigaciones como las de BalsalobreFernández (2015) y Balsalobre-Fernández et al. (2014), pero, no obstante, la relación entre ambas no tuvo significación alguna.

La explicación más lógica a esta débil relación frente a lo que cabía esperarse, seguramente esté en las ya mencionadas limitaciones del estudio, donde el no poder llevar a cabo más de dos meses de recogida de datos y con sólo cinco atletas, pueda no representar fidedignamente a la población y a los fenómenos estudiados en general. Asimismo, si añadimos las variables extrañas que no se han podido ser controladas, como las lesiones (que han sido varias y, además, de carácter importante), enfermedades, los periodos de vacaciones que coincidieron en esos dos meses y las competiciones de carácter internacional defendiendo a la selección de España (con todo lo que ello conlleva, vacunas, largos viajes, etc.), principalmente, ello sólo hace que acentuar la variabilidad que se pueda obtener en las variables registradas.

Asimismo, también se pueda deber a las condiciones climatológicas. Es decir, a pesar de que las condiciones externas cuando hacían los diferentes tipos de trabajos solían ser homogéneas y constantes en el tiempo, el factor meteorológico no puede ser controlado y está demostrado que puede afectar al rendimiento, tanto físico como mental. Por ejemplo, en el estudio de Van Cutsem, et al. (2017b) se comprobó que unas condiciones de $30^{\circ}$ C y $30 \%$ pueden aumentar la fatiga mental expresada por el propio atleta.

A razón de ello, al analizar las condiciones meteorológicas de los dos meses de recogida de datos (marzo y abril), éstos corresponden con el inicio y desarrollo de la primavera, y, por consiguiente, con el inicio de aquellos días donde el calor empieza a ser más fuerte. Y todo ello acentuado por ser la zona centro de Madrid. Además, al ser el inicio de los días calurosos del año, donde se produce una alternancia con días de también bastante frío, la adaptación de los atletas a dichas condiciones es escasa. Y es que, la aclimatación al calor es un fenómeno que requiere de la exposición repetida al calor para mantener las adaptaciones y que su efecto persista varias semanas (Redondo, 2012).

En definitiva, si a la relativamente escasa frecuencia de recogida de ciertos parámetros, se le resta las que no han podido registrarse por lesión y vacaciones, y, luego, haya ciertas recogidas de datos que, quizás, debido a la lesión y/u otros parones y variables extrañas, éstas se distorsionen debido a las desadaptaciones y/o alteraciones que generan, el resultado más probable es que las relaciones que busques, al menos, se vean mermadas. Por ejemplo, en el estudio de Liberal, Escudero, Cantallops y Ponseti (2014) se demostraron los impactos negativos que tienen las lesiones sobre el bienestar psicológico posterior a la lesión, como el estado emocional, la ansiedad cognitiva o la percepción de dominio del entorno. A razón de ello, no sería de extrañar que, todo ello tuviera una connotación negativa sobre el esfuerzo percibido que el atleta tiene sobre la carga de entrenamiento, provocando que su puntuación sea, cuanto menos, diferente a la que en situaciones de plena salud tendría.

Asimismo, se debe destacar que en los artículos de Balsalobre (2015) o Balsalobre et al. (2014a), utilizaron, en lugar del RPE CR-10, la session RPE, que, como ya se ha comentado, aúna en un mismo valor la duración e intensidad para calcular el estrés interno que ha supuesto para el atleta la sesión en general. Anteriormente, ya se habían comentado los motivos por los que no se decidió llevar este procedimiento, pero en vistas de los resultados y de su alta validez en la literatura científica, quizás sea un método muy a tener en cuenta para futuras investigaciones. 
Respecto al objetivo de la investigación, esto es, llevar a cabo un método de monitorización y cuantificación de la carga de entrenamiento que sea válido y completo, englobando información objetiva y subjetiva, así como intrínseca y extrínseca al atleta, y teniendo en cuenta el contexto que rodea a éstos, al tratarse de unos propósitos que, debido a la manera en que se ha encaminado este estudio, no se pueden demostrar de forma cuantitativa ni comparar con otros procedimientos para observar si es mejor o peor, su veracidad y demostración es mucho más complicado.

No obstante, lo que sí que se puede llevar a cabo es la utilización de la literatura científica como base que sustente y corrobore las decisiones que han hecho que en este estudio se utilice un tipo de sistema de control y seguimiento de los entrenamientos u otro.

Así pues, dadas las peculiaridades y condicionantes del lugar de prácticas, y sustentándose en la literatura científica sobre métodos para monitorizar las cargas de entrenamiento, se optó por la proposición ya citada debido a los motivos que se explicarán a continuación.

Para la cuantificación de la frecuencia, se decidió llevar un seguimiento del número de sesiones realizadas durante el periodo de intervención y, por consiguiente, de los días de descanso. Asimismo, y teniendo en cuenta que no todas las sesiones y sus respectivos contenidos tienen un mismo carácter, también se clasificó y contabilizó los diferentes tipos de sesiones por unidad de tiempo. De esta manera, se consigue ser más precisos a la hora de saber las demandas que ha supuesto una sesión y, por consiguiente, su relación con el tiempo de recuperación que necesita el atleta hasta volver a trabajar un contenido similar (Legaz-Arrese, 2012). A largo plazo, contabilizando los diferentes tipos de sesiones y sus contenidos, posibilita, entre otras cosas, el poder comparar lo trabajado entre diferentes periodos y sujetos, no sólo a nivel cuantitativo ( $\mathrm{n}^{\circ}$ de sesiones) sino también cualitativo, el hacer progresiones (por ejemplo, si un atleta venía haciendo una sesión de fuerza a la semana, y se quiere progresar en ese aspecto con él, una opción podría ser el de aumentar la frecuencia), etc.

En cuanto al volumen, la opción elegida fue la de la contabilización del número de kilómetros por unidad de tiempo, ya que, tal y como ya se ha comentado, es el indicador más recurrente para tal fin en modalidades de carrera de fondo y medio fondo, y, por ende, para expresar la carga externa de dichos deportistas (BalsalobreFernández, 2015). De igual manera, si se tiene en cuenta el nivel de los deportistas, que en el presente estudio se trata de atletas jóvenes, pero de bastante categoría, la alternativa más habitual sigue siendo la de calcular el volumen en distancia (kilómetros, principalmente) y no en tiempo, cuya última elección sería más apropiada para atletas de menos nivel (Esteve-Lanao, 2007). Asimismo, como aspecto innovador de este trabajo (al menos no se han encontrado estudios que lo lleven a cabo), se diferenció entre el número e intensidad de kilómetros llevados a cabo en los rodajes y en las partes principales de cada sesión, ya que, aunque existe relación entre ambos trabajos, muchas veces da la impresión de que conforman dos sesiones en una. De esta manera, cabe la posibilidad de diferenciar el volumen e intensidad de kilómetros en cada una de estas dos partes, así como precisar aún más a la hora de analizar y llevar un seguimiento sobre dichos aspectos. Y es que, de no realizarlo así, y dar un único valor sobre la sesión en general, dicho análisis no podría llevarse a cabo o, al menos, sería mucho más complicado.

Ligado a lo anterior, pero incluyendo, ya, el factor intensidad, se procedió a la clasificación y distribución de los kilómetros recorridos por zonas de entrenamiento. El método de las zonas de entrenamiento se puede establecer a partir de numerosos indicadores (potencia, velocidad de carrera, FC, lactato en sangre, etc.). En este caso, 
dadas las circunstancias y medios disponibles, se optó por el uso de un indicador de carga externa, como es la velocidad de carrera, ya que no hubo posibilidad de monitorizar todos los días algún parámetro de carga interna como puede ser la FC (además de que, estas últimas opciones suelen ser mucho más invasivas para el deportista). Sin embargo, lo que sí que se aprovechó y utilizó (de hecho, por ese motivo se escogió la muestra en cuestión), fueron los test de lactato progresivos realizados recientemente antes de empezar a recoger los datos, para establecer dichas zonas. De esta forma, lo que se busca es, a pesar de utilizar un indicador de carga externa, individualizar la distribución de dichos kilómetros en función de las demandas internas (en este caso, perfil de lactato y concentraciones de referencia) de cada deportista (Mujika, 2006). Y es que, los métodos de cuantificación que integran variables fisiológicas individuales ofrecen mayor consistencia en cuanto a la relación dosis-respuesta de la carga del entrenamiento (Sanders, Abt, Hesselink, Myers y Akubat, 2017). Asimismo, este procedimiento evita de tener que extraer sangre en todas las sesiones, con todo lo que ello conlleva. Además, es sabido que dicho metabolito tiene una muy estrecha relación con la intensidad y con el consumo de oxígeno (Hale, 2008). A razón de todo ello, y, en definitiva, lo que se realizó fue la individualización de las zonas de entrenamiento de cada atleta en función de la velocidad de carrera que obtuvo a determinadas concentraciones de lactato.

En cuanto al número de zonas, finalmente se introdujeron 5 , con el fin de conseguir que haya un mayor ajuste y diferenciación a la hora de controlar y cuantificar los esfuerzos. Ya que, por ejemplo, en el caso de Lucías' TRIMP (Lucía, Hoyos, Carvajal y Chicharro, 1999), éstos sólo confeccionan 3, y ello provoca que esfuerzos muy dispares, como, por ejemplo, esfuerzos por encima, ligeramente, del VT2 y los de VAM, tengan una misma contabilización (Cejuela-Anta y Steve-Lanao, 2011). En contraposición, en el caso del método ECOs desarrollado por los anteriores autores, establecen un método con muchas más zonas de entrenamiento, pero, sin embargo, su gran problemática reside en la cierta subjetividad que siempre se asume a la hora de establecer las diferentes zonas y que, por lo tanto, no se ajuste, verdaderamente, a las demandas internas del deportista. De tal manera que, a mayor número de zonas de entrenamiento, mayor subjetividad y riesgo se asume, lógicamente. Por todo ello, se decidió coger este sistema de 5 zonas de entrenamiento, los cuales cubren toda la curva de intensidad y, además, recogen las principales umbrales y límites de referencia.

Asimismo, destacar que, para un mayor detalle de los kilómetros recorridos y su intensidad (velocidad de carrera), y aprovechando que todos ellos contaban con algún weareble (en concreto relojes) que incluyen GPS, se utilizó dicha tecnología para tal registro. Pues no se debe olvidar que, una cosa es lo que el entrenador programa y prescribe y otra lo que el atleta lleva a cabo (Mujika, 2006), y muchas veces difiere notablemente. Por otro lado, también es verdad que, dicha precisión puede sufrir variaciones. En este sentido, se ha demostrado (y la experiencia de los atletas lo corroboran) que la contabilización de los metros recorridos en pista mediante GPS, en numerosas ocasiones, varía, y cómo dicha contabilización es más fácil y fiable (contando las vueltas que ha hecho cada atleta, o multiplicando el número de series por la distancia programada por el entrenador, etc.), en estos casos, se optó por apuntar los kilómetros y su ritmo manualmente. Es decir, el uso del GPS se usó, sobre todo, para la monitorización de los rodajes, los cuáles son más difíciles de controlar en cuanto a volumen e intensidad ya que se solían realizan por bosque y/o ciudad.

Por otra parte, pero siguiendo con la variable intensidad, se decidió usar también para su monitorización, pero desde el punto de vista de la carga interna, el esfuerzo percibido, mediante la Escala de Esfuerzo Percibido (RPE), y, en concreto, la Escala RPE CR-10 (Borg, 1982; Borg, 1990), cuya numeración para expresar la intensidad va de 0 (reposo) a 10 (máximo) (ver Tabla 1). Y es que a esta escala de 
valores suelen estar más familiarizados los deportistas que la que va de 6 a 20 (RPE 6-20) (que se trata de la otra más conocida al respecto).

Dicho lo cual, la elección y uso de este método subjetivo se fundamenta en varias razones. La primera, es su amplia demostración científica de fiabilidad y validez (Borresen y Lambert, 2008; Borresen y Lambert, 2009; Lambert y Borresen, 2010). Y es que, las medidas subjetivas pueden llegar a reflejar cargas de entrenamiento agudas y crónicas con mayor sensibilidad y consistencia que determinadas medidas objetivas (Saw, Main y Gastin, 2016). La segunda, es su facilidad de poner en práctica, debido a su sencillez, rapidez, cero costes y su prácticamente nula interferencia en el entrenamiento de los atletas. La tercera, su capacidad para mostrar un valor individualizado sobre la intensidad del entrenamiento y poder monitorizar esfuerzos físicos que mediante la velocidad de carrera no es posible, como con el trabajo de fuerza (Cejuela-Anta y Esteve-Lanao, 2011). Y la cuarta, y no menos importante, se debe a que, desde el punto de vista de los realizadores de este trabajo, y tal y como se puede apreciar en la literatura científica, son cada vez más los datos (principalmente, objetivos) con los que los deportistas, entrenadores, preparadores físicos, etc. pueden monitorizar los esfuerzos físicos, lo cual, por una parte, está bien, pues es más información con la que poder ajustar todo este proceso, pero, sin embargo, todo este boom de datos está generando una casi total dependencia hacia éstos, lo que hace que, además de, muchas veces, no saber utilizar y encajar todos ellos, está provocando que los deportistas estén olvidando algo tan sencillo y a la vez tan útil como es controlar sus esfuerzos según sus propias sensaciones. Es decir, dicha capacidad de autorregulación se está perdiendo debido a una mayor dependencia tecnológica, la cual, en la gran mayoría de casos, no tiene en cuenta una serie de aspectos internos del deportista que, quizás, se puedan considerar como secundarios en el proceso del entrenamiento, pero que hoy en día sabemos que tiene una gran importancia a la hora de lograr el máximo rendimiento. Se está haciendo alusión a aspectos como la falta de sueño y/o nutrición, factores psicológicos, emocionales, o cualquier condicionante de la vida personal del deportista que pueden hacer que la capacidad de éste decaiga y sea conveniente, por consiguiente, adaptar el entrenamiento según sus percepciones a fin de entrenar mejor y/o evitar problemas mayores, tales como sobrecargas no funcionales, lesiones y/o sobreentrenamientos.

Asimismo, no se debe olvidar que, las carreras de fondo y medio fondo se encajan dentro de las modalidades de resistencia, lo que conlleva realizar, tanto en los entrenamientos como en la competición, esfuerzos prolongados en el tiempo. Esto supone que, en numerosas ocasiones, la intensidad, y, sobre todo, la duración de los esfuerzos aumente la fatiga mental de los deportistas, lo cual suele acarrear una disminución del rendimiento ligado a una mayor percepción del esfuerzo (Van Cutsem et al., 2017a). Ello nos refleja la importancia de tener en cuenta la carga mental en los deportistas en general, y en los de resistencia en especial, ya que, siguiendo a estos mismos autores, es en los trabajos que se dilatan en el tiempo donde la fatiga mental más influye, mientras que, en los esfuerzos anaeróbicos, de fuerza máxima y/o potencia, dicho fenómeno no afecta.

Por todo ello, se preguntó al deportista en cada entrenamiento sobre lo intenso que le había parecido éste, siendo 1 "Muy, muy cómodo" y 10 "Máximo" y 0 en caso de reposo total. Asimismo, se pidió al atleta que diferenciara dos valores dentro del mismo entrenamiento, uno haciendo alusión, únicamente, al rodaje y otro a la sesión en general. Todo ello en pos de poder comprobar la carga interna que supone al deportista la realización de los rodajes (en caso de que lo hiciera, pues hay alguna excepción en la que no, por ejemplo al tener ciertas lesiones), así como poder comparar el estrés interno que supone la intensidad (velocidad) a la que se hacen los kilómetros recorridos (carga externa), ya que los rodajes suelen ser de mayor volumen pero intensidad baja (Zona 1, en su gran mayoría) y los kilómetros recorridos en la 
parte principal de la sesión (en caso de que toque ese tipo de trabajo) suelen ser de menor volumen pero, sin embargo, de mucha más intensidad (en una zona u otra en función de lo que quiera trabajar el entrenador).

Por último, se midió semanalmente la altura en el salto vertical, en concreto, en el CMJ, con el objetivo de comprobar la fatiga neuromuscular acumulada que puede generarse en función del volumen total de kilómetros recorridos, la intensidad de éstos según su distribución por zonas de entrenamiento, la frecuencia de trabajo de los diferentes esfuerzos y en relación con los valores medios de RPE. Y es que, en el fenómeno de la fatiga, sea cual sea su causa, en última instancia lo que provoca es una disminución para generar fuerza (Allen at al., 2008). Consecuentemente, dicho procedimiento se ha validado como adecuado para tales fines, tal y como nos demuestran investigaciones como las de Balsalobre-Fernández et al. (2014) 0 Petersen, Hansen, Aagaard y Madsen (2007) y, además, a diferencia de otros métodos, es barato, sencillo, no interfiere en los entrenamientos y es poco invasivo en el atleta (Buchheit y Laursen, 2013; Jiménez-Reyes et al., 2011). Además, para tal fin lo único que se necesitó fue la aplicación de móvil MyJump, por lo que su coste y puesta en escena fue, prácticamente, nulo, y como ya se ha comentado, cuenta con validación científica (Balsalobre-Fernández et al., 2015).

Finalmente, en relación a las aplicaciones de móvil, destacar otra tipología que, a pesar de no ser propia del mundo del deporte, pueden ser de gran ayuda a la hora de llevar a cabo el proceso del seguimiento del entrenamiento y sus cargas. Se está haciendo alusión a las aplicaciones de mensajería instantánea y/o redes sociales, las cuales te permiten, como el propio nombre indica, poder relacionarte con otras personas de manera gratuita, rápida y clara. En este caso, se optó por la utilización de Whatsapp, ya que lo que se buscaba era, únicamente, saber, en los días en los que no coincidían ambas partes interesadas (atletas e investigador), cualquiera de las variables citadas (excepto, lógicamente, el CMJ, donde siempre debían coincidir ambas partes).

En definitiva, con todo este sistema de monitorización y cuantificación de la carga de entrenamiento, lo que se ha buscado en un método que, teniendo en cuenta, como ya se ha explicado anteriormente, la modalidad deportiva, el tipo de deportistas y los medios al alcance, integre tanto parámetros de carga externa como interna, pues su combinación puede ser la clave que ayude a optimizar el desarrollo del atleta (Mujika, 2017). De esta manera, mediante su correlación se puede llegar a revelar estados de fatiga en el deportista, tal y como nos indican autores de la talla de Halson (2014) y, por consiguiente, se trata de la mejor opción de monitoreo, pues ello puede contribuir a optimizar el desarrollo del deportista, a detectar a tiempo estados de sobrecarga no funcional o sobreentrenamiento y a reducir las lesiones (Roos, Taube, Brandt, Heyer, y Wyss, 2013).

\section{Limitaciones y futuras líneas de investigación}

En primer lugar, el aspecto cuantitativo de la muestra, pues a pesar de ser de un alto nivel cualitativo (todos ellos han representado a España en campeonatos internacionales), sólo se ha podido contar con cinco unidades muestrales. Consecuentemente, sería conveniente, para futuras investigaciones, contar con un mayor número de participantes. De igual manera, el incluir otras variables en la muestra, como el sexo o la edad, para ver si existen diferencias entre dichos grupos, podría tener su cabida. Por último, dado que existe poca literatura científica al respecto (principalmente, respecto a la medición de la fatiga neuromuscular mediante la altura del CMJ), podría ser interesante aplicar dicho método en otras modalidades, tanto dentro del atletismo, como en otros deportes (lógicamente, siempre y cuando, la fatiga neuromuscular del tren inferior exista en éstos). 
A razón de ello, en vistas de futuro, sería favorable ampliar dicho periodo temporal y poder hacer comparaciones de cualquiera de dichas variables estudiadas a lo largo de una etapa competitiva o, incluso, entre diferentes temporadas. Por ejemplo, un tema que cada vez está más en auge es el de afinamiento o tapering antes de una competición. Así pues, se podría comprobar las modificaciones en cuanto a la carga de entrenamiento (tanto interna como externa) entre periodos competitivos de mayor 0 menor importancia, así como el grado de fatiga (y descanso) del atleta.

Asimismo, intentando subsanar estas dos limitaciones (cantidad de unidades muestrales y tiempo de recogida de datos), también se conseguirá que las variables extrañas que puedan ir surgiendo a lo largo de la investigación (lesiones, enfermedades, periodos de vacaciones, condiciones climatológicas, viajes, etc.) tengan un menor peso y, por consiguiente, alteren en menor medida los resultados.

\section{CONCLUSIONES}

- La monitorización de las cargas de entrenamiento se trata de un proceso trascendental. Ahora bien, tan negativo resulta no cuantificar, como cuantificar mal. De entre la carga planificada, prescrita y realizada (Mujika, 2006), se debe quedar, al menos, con la última. Asimismo, parece ser que el mejor sistema de monitorización es aquel que combina parámetros tanto de carga externa como interna, así como objetivos y subjetivos. De esta manera, correlacionando la carga externa e interna se puede deducir el grado de fatiga (o descanso) del atleta (Halson, 2014).

- Existen multitud de indicadores, sistemas y métodos de monitorización y cuantificación de la carga. Ninguno ha sido considerado, hasta la fecha, como el ideal, pues todos ellos cuentan con sus ventajas e inconvenientes. Por todo ello, en función de la modalidad deportiva, del tipo de deportista y de los medios al alcance, el entrenador y/o preparador físico deberá sopesar cuáles les es más eficientes.

- Para la cuantificación de la carga externa en atletas de fondo y medio fondo de alto nivel, la utilización del número de kilómetros por unidad de tiempo, las zonas de entrenamiento por velocidad de carrera a partir de los umbrales y perfil individual de lactato y la frecuencia de sesiones así como de los diferentes tipos de contenidos de trabajo, permite llevar un seguimiento de las principales variables que influyen en la carga de entrenamiento (volumen, intensidad, frecuencia y naturaleza de los contenidos), pudiendo hacer comparaciones intra e intersujetos.

- En cuanto al control y seguimiento de la carga interna, la Escala de Esfuerzo Percibido (RPE CR-10) demuestra ser un método válido, fiable, fácil, rápido, barato y poco invasivo en el atleta. En este estudio corroboró una relación significativa con el número de kilómetros (volumen) total y a diferentes intensidades (zonas de entrenamiento). Respecto a la intensidad en sí misma, se obtuvo una relación positiva, aunque no significativa (debido, seguramente a las limitaciones del estudio), cada vez más fuerte a medida que se aumentaba la intensidad. Asimismo, el RPE de la sesión (session RPE) puede que sea también una alternativa muy a tener en cuenta para calcular el estrés interno de una sesión en general (volumen por intensidad).

- Con todo, para la comparación de la carga interna que supone los diferentes tipos de contenidos de trabajo en atletas de fondo y medio fondo, se debe tener en cuenta que, depende, en gran medida, no sólo de la naturaleza de dichos esfuerzos, sino también del volumen e intensidad con que se lleven a cabo. Ello provoca que haya una gran variabilidad en cuanto a carga interna en función de 
dichas variables. Consecuentemente, salvo que se mantengan constantes (que no es lo habitual, debido a los principios del entrenamiento, tales como el principio de variabilidad, del incremento progresivo de la carga, etc.), quizás, lo mejor sea comparar dicho estrés interno, únicamente, entre mismos contenidos.

- En cuanto al proceso de la fatiga, la pérdida de altura en el CMJ puede ser un método válido y fiable para su control desde el punto de vista neuromuscular. Asimismo, su poco coste, su rapidez y su escaso grado de invasión en el atleta y en su proceso de entrenamiento, lo hacen un mecanismo ideal para poder realizarlo de manera frecuente. En este caso, demostró ser un método acorde para la cuantificación de la fatiga subaguda (semanal), teniendo una relación significativa con el volumen (número de kilómetros), la intensidad (zonas de entrenamiento por velocidad de carrera a partir de los umbrales y perfil individual de lactato) e, incluso, la frecuencia de algunos tipos de contenidos, como las series.

\section{REFERENCIAS}

Allen, D. G., Lamb, G. D., y Westerblad, H. (2008). Skeletal muscle fatigue: cellular mechanisms. Physiological Reviews, 88(1), 287-332.

Balsalobre-Fernández, C. (2015). Monitorización y estudio de las relaciones entre la carga de entrenamiento, la producción de fuerza, la fatiga y el rendimiento en corredores de alto nivel (Tesis doctoral). Universidad Autónoma de Madrid.

Balsalobre-Fernández, C., Glaister, M., y Lockey, R. A. (2015). The validity and reliability of an iPhone app for measuring vertical jump performance. Journal of Sports Sciences, 33(15), 1574-1579.

Balsalobre-Fernández, C., Tejero-González, C. M., y del Campo-Vecino, J. (2014). Relationships between training load, salivary cortisol responses and performance during season training in middle and long distance runners. PLoS One, 9(8), 1-6.

Banister, E. W., y Calvert, T. W. (1980). Planning for future performance: implications for long term training. Canadian Journal of Applied Sport Sciences. Journal Canadien des Sciences Appliquées au Sport, 5(3), 170.

Billat, V. (2002). Fisiología y metodología del entrenamiento. De la teoría a la práctica (Vol. 24). Paidotribo.

Borg, G. A. (1982). Psychophysical bases of perceived exertion. Med Sci Sports Exerc, 14(5), 377-381.

Borg, G. (1990). Psychophysical scaling with applications in physical work and the perception of exertion. Scandinavian Journal of Work, Environment \& Health, 16(1), 55-58.

Borresen, J., y Lambert, M. I. (2008). Quantifying training load: a comparison of subjective and objective methods. International Journal of Sports Physiology and Performance, 3(1), 16-30.

Borresen, J., y Lambert, M. I. (2009). The quantification of training load, the training response and the effect on performance. Sports Medicine, 39(9), 779-795. 
Bourdon, P. C., Cardinale, M., Murray, A., Gastin, P., Kellmann, M., Varley, M. C., ... y Cable, N. T. (2017). Monitoring Athlete Training Loads: Consensus Statement. International Journal of Sports Physiology and Performance, 12(2), 161-170.

Buchheit, M., y Laursen, P. B. (2013). High-intensity interval training, solutions to the programming puzzle. Part II: anaerobic energy, neuromuscular load and practical applications. Sports Medicine, 43(5), 313-338.

Casajús, J. A., Piedrafita, E., y Aragonés, M. T. (2009). Criterios de maximalidad en pruebas de esfuerzo. Revista Internacional de Medicina y Ciencias de la Actividad Física y del Deporte, 9(35), 217-231.

Cejuela-Anta, R., y Esteve-Lanao, J. (2011). Training load quantification in triathlon. Journal of Human Sport \& Exercise, 6(2), 218-232.

Coggan, A. R. (2003). Training and racing using a power meter: an introduction. Site Inspired performance multisports (en línea). http://www.inspiredperformancecoaching.com/ref lib/Coggan Power Meter.pdf . Página consultada el 16 de marzo del 2017.

Esteve Lanao, J. (2007). Periodización y control del entrenamiento en corredores de fondo (Tesis doctoral). Universidad Europea de Madrid.

Fernández-del-Olmo, M., Rodriguez, F. A., Marquez, G., Iglesias, X., Marina, M., Benitez, A., ... y Acero, R. M. (2013). Isometric knee extensor fatigue following a Wingate test: peripheral and central mechanisms. Scandinavian Journal of Medicine \& Science in Sports, 23(1), 57-65.

Fitts, R. H. (1994). Cellular mechanisms of muscle fatigue. Physiological Reviews, 74(1), 49-94.

Foster, C. (1998). Monitoring training in athletes with reference to overtraining syndrome. Medicine and Science in Sports and Exercise, 30, 1164-1168.

Hale, T. (2008). History of developments in sport and exercise physiology: AV Hill, maximal oxygen uptake, and oxygen debt. Journal of Sports Sciences, 26(4), $365-400$.

Halson, S. L. (2014). Monitoring Training Load to Understand Fatigue in Athletes. Sports Med, 44(Suppl 2): 139-147.

Imaz, I. A. (2013). Comparación de diferentes métodos para el cálculo del umbral anaeróbico individual y su equivalencia con el máximo estado estable (Tesis Doctoral). Universidad del País Vasco.

Jiménez-Reyes, P., Cuadrado-Peñafiel, V., y González-Badillo, J. J. (2011). Aplicación del CMJ para el control del entrenamiento en las sesiones de velocidad. Cultura, ciencia y deporte: Revista de Ciencias de la Actividad Física y del Deporte de la Universidad Católica de San Antonio, 6(17), 105-112.

Lambert, M. I., y Borresen, J. (2010). Measuring training load in sports. International Journal of Sports Physiology and Performance, 5(3), 406-411.

Legaz-Arrese, A. (2012). Manual de entrenamiento deportivo. Barcelona: Paidotribo. 
Liberal, R., Escudero, J. T., Cantallops, J. y Ponseti, J. (2014). Impacto psicológico de las lesiones deportivas en relación al bienestar psicológico y la ansiedad asociada a deportes de competición. Revista de Psicología del Deporte, 23(2), 451-456.

Lucía, A., Hoyos, J., Carvajal, A., y Chicharro, J. L. (1999). Heart rate response to professional road cycling: the Tour de France. International Journal of Sports Medicine, 20(03), 167-172.

Manzi, V., Bovenzi, A., Castagna, C., Salimei, P. S., Volterrani, M., y lellamo, F. (2015). Training-load distribution in endurance runners: Objective versus subjective assessment. International Journal of Sports Physiology and Performance, 10(8), 1023-1028.

Mujika, I. (2006). Métodos de cuantificación de las cargas de entrenamiento y competición. Kronos: Revista Universitaria de la Actividad Física y el Deporte, 5(10), 1-10.

Mujika, I. (2010). Intense training: the key to optimal performance before and during the taper. Scandinavian Journal of Medicine \& Science in Sports, 20(s2), 24-31.

Mujika, I. (2013). The alphabet of sport science research starts with Q. International Journal of Sports Physiology and Performance, 8, 465-466.

Mujika, I. (2017). Quantification of Training and Competition Loads in Endurance Sports: Methods and Applications. International Journal of Sports Physiology and Performance, 12(2), 9-17.

Mujika, I., Goya, A., Ruiz, E., Grijalba, A., Santisteban, J., y Padilla, S. (2002). Physiological and performance responses to a 6-day taper in middle-distance runners: influence of training frequency. International Journal of Sports Medicine, 23(05), 367-373.

O'Connor, P. J. (2007). Monitoring and titrating symptoms. Sports Medicine, 37(4-5), 408-411.

Pageaux, B. (2016). Perception of effort in exercise science: definition, measurement and perspectives. European Journal of Sport Science, 16(8), 885-894.

Pallarés, J. G. y Morán-Navarro, R. (2012). Propuesta metodológica para el entrenamiento de la resistencia cardiorrespiratoria. Journal of Sport and Health Research, 4(2), 119-136.

Petersen, K., Hansen, C. B., Aagaard, P., y Madsen, K. (2007). Muscle mechanical characteristics in fatigue and recovery from a marathon race in highly trained runners. European Journal of Applied Physiology, 101(3), 385-396.

Petibois, C., Cazorla, G., Poortmans, J. R., y Déléris, G. (2002). Biochemical aspects of overtraining in endurance sports. Sports Medicine, 32(13), 867-878.

Redondo, R. B. (2012). Aclimatación al ejercicio físico en situaciones de estrés térmico. Archivos de Medicina del Deporte: Revista de la Federación Española de Medicina del Deporte y de la Confederación Iberoamericana de Medicina del Deporte, 29(148), 621-631. 
Roos, L., Taube, W., Brandt, M., Heyer, L., y Wyss, T. (2013). Monitoring of daily training load and training load responses in endurance sports: what do coaches want. Schweizerische Zeitschrift für Sportmedizin \& Sporttraumatologie, 61(4), 30-36.

Sanders, D., Abt, G., Hesselink, M.K., Myers, T., y Akubat, I. (2017). Methods of Monitoring Training Load and Their Relationships to Changes in Fitness and Performance in Competitive Road Cyclists. International Journal of Sports Physiology and Performance, 17, 1-23.

Saw, A. E., Main, L. C., y Gastin, P. B. (2016). Monitoring the athlete training response: subjective self-reported measures trump commonly used objective measures: a systematic review. British Journal of Sports Medicine, 50, 281-291.

Tuimil, J. L., Iglesias, E., Dopico, X., y Morenillas, L. (2005). Efectos del entrenamiento continuo e interválico de carga externa similar sobre la frecuencia cardiaca. European Journal of Human Movement, 13, 107-118.

Van Cutsem, J., Marcora, S., De Pauw, K., Bailey, S., Meeusen, R., y Roelands, B. (2017a). The Effects of Mental Fatigue on Physical Performance: A Systematic Review. Sports Medicine, 1-20. Recuperado de https://www.researchgate.net/publication/312036806 The Effects of Mental F atigue on Physical Performance A Systematic Review

Van Cutsem, J., De Pauw, K., Buyse, L., Marcora, S. M., Meeusen, R., y Roelands, B. (2017b). Effects of Mental Fatigue on Endurance Performance in the Heat. Medicine and Science in Sports and Exercise. Recuperado de https://www.researchgate.net/publication/314654941 Effects of Mental Fatigu e on Endurance Performance in the Heat

Wallace, L. K., Slattery, K. M., y Coutts, A. J. (2009). The ecological validity and application of the session-RPE method for quantifying training loads in swimming. The Journal of Strength \& Conditioning Research, 23(1), 33-38.

Wallace, L. K., Slattery, K. M., Impellizzeri, F. M., y Coutts, A. J. (2014). Establishing the criterion validity and reliability of common methods for quantifying training load. The Journal of Strength \& Conditioning Research, 28(8), 2330-2337. 